\title{
Filigrane
}

Écoutes psychothérapiques

\section{Présentation. Psychanalyse et engagement II}

\section{Sophie Gilbert et Véronique Lussier}

Volume 21, numéro 2, automne 2012

URI : https://id.erudit.org/iderudit/1015194ar

DOI : https://doi.org/10.7202/1015194ar

Aller au sommaire du numéro

Éditeur(s)

Revue Santé mentale au Québec

ISSN

1192-1412 (imprimé)

1911-4656 (numérique)

Découvrir la revue

Citer ce document

Gilbert, S. \& Lussier, V. (2012). Présentation. Psychanalyse et engagement II.

Filigrane, 21(2), 7-10. https://doi.org/10.7202/1015194ar d'utilisation que vous pouvez consulter en ligne.

https://apropos.erudit.org/fr/usagers/politique-dutilisation/ 


\section{Présentation. Psychanalyse et engagement II}

\section{Sophie Gilbert et Véronique Lussier}

$\mathrm{D}$ ans ce second volet (voir la première partie dans le vol. $21, \mathrm{n}^{\circ} 1$ ) de notre dossier sur l'engagement en psychanalyse, nous avons le plaisir d'accueillir une diversité de points de vue sur la psychanalyse engagée. Qu'il s'agisse de l'implication du clinicien au sein d'une équipe institutionnelle, de la posture de la psychanalyse face à l'engagement du sujet dans la collectivité, de l'engagement mutuel dans une démarche thérapeutique, mais aussi du rôle particulier du clinicien-chercheur d'allégeance psychanalytique dans la compréhension de problématiques socioculturelles, tous nos auteurs témoignent de la vitalité d'une psychanalyse bien ancrée (engagée?) dans l'actualité. Se rajoute à cette tonalité de l'engagement celle, plus pragmatique peut-être, de cliniciens qui œuvrent dans différents milieux d'intervention témoignant autant de la pratique en institution que de celle en milieu communautaire. Ce faisant, ils se confrontent à la nécessité d'imaginer de nouvelles modalité du cadre qui tiennent compte de la complexité des problématiques d'aujourd'hui, des savoirs issus d'autres champs épistémologiques, et ce, sans trahir cette discipline, et surtout, sans sombrer dans l'utopique abolition de tout reste (Lévy).

Comment se fait-il que cette psychanalyse, que ces cliniciens, qui prennent position dans les institutions, dans les communautés, ou par la force de leur plume, puissent cohabiter avec une image sociétale de la psychanalyse dépassée, isolée, vieillissante? Y aurait-il une faille au plan de la diffusion de cette approche, des échanges autour de celle-ci, et surtout, de l'accessibilité de ces échanges dans les milieux cliniques moins spécifiques, et dans le social?

D’un continent à l'autre, du Nord au Sud, ce numéro ravira ceux qui voient en l'engagement de la psychanalyse une véritable ouverture. Ouverture à d'autres disciplines, à d'autres cultures, à d'autres races, mais aussi, au socius comme partie intégrante du sujet. Autant d'auteurs que de points de vue, donc, dans ce second numéro. Foisonnement d'idées, de perspectives, et de diverses expressions du flirt (au sens pulsionnel d'Éros, du lien) de la 
psychanalyse avec d'autres disciplines. La psychanalyse n'a-t-elle pas été ainsi conçue? En effet, la rigueur et la transparence des écrits freudiens démontrent bien la pertinence de cet échange, de ce dialogue entre la médecine, la philosophie, la littérature, l'anthropologie, etc., dans le processus de création, de la naissance (Bossé) à l'évolution constante d'une psychanalyse... engagée.

Notre dossier s'amorce avec une réflexion illustrée par une situation clinique complexe, relative à l'intervention psychosociale institutionnelle de nos services de protection de l'enfance. L'humilité de l'auteure, Lorraine Boucher, fera réfléchir tout clinicien sur les enjeux inhérents à son propre engagement auprès de ses analysants, clients ou patients. Quelle place estelle laissée à l'autre: analysant, consultant, mais aussi, aux différents partenaires de l'intervention clinique? Dans la situation présentée, l'engagement de l'analyste tient véritablement à l'espace accordé au déploiement d'un matériel de travail - à potentiel analytique - chez la famille, à l'aide des intervenants sociaux. Ici se dévoile le soutien inébranlable d'une psychanalyste, afin d'instaurer puis de protéger un espace de parole même dans les situations apparemment les plus réfractaires à cette approche.

Dans un certain recul par rapport au quotidien de la clinique, Roland Gori, avec le talent argumentatif que nous lui connaissons, offre une véritable dissertation à partir de l'historique du thème à l'honneur, pour ensuite aborder l'influence de l'environnement socioculturel sur le savoir et les pratiques, dont la pratique clinique bien sûr. Dans ce contexte, l'engagement de l'individu se fait au détriment de sa liberté, voire de ses possibilités de réelle émancipation, et parfois même, de subjectivation. Pourtant, la psychanalyse, en porte-à-faux avec cette domination d'un certain savoir, d'un certain pouvoir aussi, ne peut que faire valoir les vertus du récit et de la pensée, de même qu'une autre modalité de guérison, soit un premier pas vers l'émancipation du sujet. Il pourrait s'agir ici d'une élaboration théorique à partir de l'article précédent, ou d'une magnifique introduction à tous les cadres d'intervention présentés par la suite, dans lesquels la psychanalyse se met au service et s'installe, voire s'engage dans des milieux hétérogènes au cadre de la cure classique, mais surtout, se pose à contre-courant de certains mouvements socioculturels dominants.

Pierre Joly nous présente ensuite la spécificité et l'originalité du travail psychanalytique de groupe en milieu communautaire. L'auteur y discute principalement de l'engagement des participants, de la modulation de leur posture initiale, vers une certaine dynamique de groupe qui fait image. Comment ce groupe est-il intégré par chacun comme objet, à la fois fantasmatique et réel? 
Une illustration clinique permet d'amorcer une réponse à cette complexe question. Et une interrogation s'impose: à quoi s'engagent ce groupe, et en particulier, son meneur-analyste envers chacun des participants? Lourd mandat... de part et d'autre.

Dans la foulée de l'article précédent, relativement à l'originalité de l'implication de psychanalystes québécois dans la communauté, Isabelle Lasvergnas nous présente les premiers constats résultant des cinq ans d'existence de la Clinique psychanalytique de Montréal, une initiative de la Société psychanalytique de Montréal. L'engagement de psychanalystes à desservir une population plus vulnérable au niveau socioéconomique les a amenés à penser un cadre approprié pour ce faire. Non seulement s'agit-il d'évaluer la pertinence d'une analyse pour les consultants mais aussi, cette implication les invite à remettre en chantier des considérations cliniques fondamentales, telles les indications générales de l'analyse, les remaniements souhaitables ou non du cadre analytique, de même que l'ouverture à l'actualité de la souffrance psychique et de ses modalités.

Pour clore ce dossier, Malika Mansouri et Marie Rose Moro abordent un champ clinique très spécifique, celui de l'ethnopsychiatrie, au sein duquel la psychanalyse ne peut que s'inscrire en complémentarité avec d'autres disciplines, en premier lieu l'anthropologie. Par une recherche menée auprès de jeunes adultes français d'origine algérienne, le cumul de différents angles d'analyse permet d'affiner la compréhension psychanalytique d'événements tels que les émeutes de 2005 dans les banlieues parisiennes. Le clinicien ne peut que se sentir interpellé en tant que partie intégrante d'un système socioculturel complexe et trop souvent conflictuel, et plus largement, dans son rapport à l'altérité (notamment, à l'autre culturel).

À la suite de ce dossier, le lecteur pourra découvrir le texte de deux entrevues menées au printemps dernier, à Montréal, auprès de psychanalystes européens de passage sur notre continent. Nous souhaitons remercier tout particulièrement monsieur Ghyslain Lévy, qui se souviendra que notre thématique de l'engagement en psychanalyse est justement née d'une conversation des plus animées que nous avons eu la chance d'avoir lors d'un précédent séjour. Cette thématique ressort naturellement, en filigrane, tout au long de cette entrevue habilement menée par Ellen Corin. L'on pourrait dire que c'est ici de l'engagement de la psychanalyse, comme discipline, qu'il est d'abord question. S'engager à l'encontre de la domination d'une pensée occidentale potentiellement aliénante pour le sujet. Là où le sujet risque d'être réduit en fonction d'idéaux d'unicité (unité?), de communauté de pensée, de référents 
technologiques et scientifiques, bref, de tout ce qui abolit le reste, l'inconnu, l'insaisissable.

Une seconde entrevue retrace le parcours d'écriture de Jean-Michel Quinodoz, par l'entremise des questions de France Senécal. L'engagement de cet auteur envers la psychanalyse est palpable, de même que son grand souci de transmission d'un savoir sans cesse en évolution. Psychanalyste-écrivain, monsieur Quinodoz nous présente son ouvrage Lire Freud, lequel condense à merveille cette transmission, en évoquant le passage de son propre séminaire de lecture des textes freudiens, à un canevas didactique qui soutient aujourd'hui le travail de nouvelles générations de cliniciens. À travers l'évocation de ses expériences d'édition et de rédaction, monsieur Quinodoz dévoile les dessous d'un processus de création soutenu par la rencontre avec des psychanalystes incontournables... La création est abordée ici sous différents angles: en clinique, soutenue notamment par le rêve, puis dans les œuvres artistiques, et surtout, en tant que vecteur de vie.

La rubrique «Heteros » de ce numéro nous ramène du côté de l'engagement de la psychanalyse, cette fois entendue comme une façon de se lier, de s'engager donc, auprès d'autres disciplines, telles la littérature et la philosophie. Dans un texte très personnel, «signé», Michel Peterson propose une analyse informelle de Nelly (Arcan), là où l'auteure se confond avec le principal personnage de son œuvre. Curieusement, ici, l'auteur-clinicien semble laisser toute la place à celle qui a déjà tout dit, au travers de son œuvre... du moins, lorsque l'on se donne la peine de mettre en ordre les morceaux du puzzle, aussi habilement que l'aura fait Monsieur Peterson.

Ce numéro s'achève avec un texte d'envergure, un texte qui en soi constitue une référence, à lire et relire afin d'en saisir toutes les subtilités. Cet article, généreusement offert par Jean Bossé, retrace les éléments fondateurs de deux disciplines majeures, la philosophie et la psychanalyse, à partir de l'évocation en parallèle de deux scènes transférentielles. L'auteur rapporte donc avec moult références les éléments fondamentaux de la relation établie entre Freud et Fliess, puis celle de Platon et de Socrate. Ce faisant, il dévoile peu à peu ce qui s'avère constituer les ingrédients du passage de l'amour (au sens d'Éros) à la création d'une nouvelle discipline.

Pour clore ce numéro, la rubrique «Échos» donne la parole à Dinara Machado Guimarães qui nous emporte vers le Sud avec une brève analyse d'un film brésilien : L'année où mes parents sont partis en vacances. Ici, c'est à la rencontre du cinéma, et au travers de celui-ci, du contexte sociopolitique ambiant que se porte la psychanalyse engagée. 